\title{
Rethinking Collaborative Learning Through Participation in an Interdisciplinary Research Project: Tensions and Negotiations as Key Points in Knowledge Production
}

\author{
Valérie Tartas • Nathalie Muller Mirza
}

Published online: 8 September 2007

(C) Springer Science + Business Media, LLC 2007

\begin{abstract}
As social and cultural psychologists of learning, we are persuaded of the crucial role of interaction in development and learning. But how do we experience this assumption in our own research practices and in our collaboration with colleagues? Taking as our object of study our own participation in a European Research and Development project that aimed to enhance interactive and argumentative skills in learning settings, this study shows how collaboration among project partners is not something that is to be taken for granted, but something that is elaborated and evolves in time, takes diverse forms, and is mediated by multiple tools. The psychological processes - more particularly tensions and negotiationinvolved in collaboration are developed and discussed. The study explores the processes of establishing collaboration and, through the analysis of specific zones of tensions, sheds light on the way new knowledge (on how to do research, how to communicate, how to work together) is constructed. It contributes to the understanding of the issues and conditions for the development of a community of practice.
\end{abstract}

Keywords Collaboration · Negotiation · Tensions $\cdot$ Teamwork .

Knowledge production

Collaborative practices are a core facet of professional life. Nowadays, distributed knowledge and complex division of work make necessary the creation of networks

\footnotetext{
V. Tartas $(\bowtie)$

University of Toulouse 2, Octogone-ECCD EA no. 4156, Pavillon de la recherche,

5 allées Antonio Machado, 31058 Toulouse Cedex 09, France

e-mail: tartas@univ-tlse2.fr

N. Muller Mirza

Institut de Psychologie et Education, University of Neuchâtel, Espace Louis Agassiz 1,

2000 Neuchâtel, Switzerland 
of teams across countries and professional expertise. Moreover, information technology allows team work spread out in space and time. In such contexts, diversity becomes a key notion; diversity of people, according to their national, cultural, linguistic, professional backgrounds, and diversity of tools mediating their communication. Diversity is a phenomenon that one observes, it is a concrete fact. What about its consequences in terms of construction of knowledge?

Studies on collaboration are divided on this point: while some authors emphasize the potential of diversity to be a resource for new understandings (Akkerman et al. 2006; Derry et al. 1998), others claim that diversity is rather a constraint and a barrier to team performance and team coordination (Marks et al. 2002). Other studies in working or learning contexts, using theoretical tools from a dialogical field of research, seek not to understand whether diversity promotes or hinders the development of knowledge, but rather how meanings and knowledge are created, negotiated and co-constructed among diverse actors (Muller Mirza 2005; Wertsch and Toma 1995). It is this dialogical perspective on the significance of diversity in collaboration that we wish to advance in the present paper. The research tradition that examines whether collaboration has a productive or inhibiting effect on the production of knowledge takes the collaboration itself for granted. However, building a relation of collaboration is a complicated process. In this paper, we take a step back to look at the process of establishing collaboration, for this is fundamental to understanding the conditions of creating or not creating new knowledge in collaboration.

As developmental and cultural psychologists, we would like to create a dialogue between two kinds of experiences we have as researchers: the one we have when we use our theoretical tools for understanding the socio-psychological processes at stake in interaction learning settings; and the other we have when we are ourselves actors in collaborative interactions in an interdisciplinary research project. $\mathrm{We}^{1}$ therefore take our participation in such a project as a point of departure for exploring the socio-cultural dynamics underlying collaborative research. We also take this opportunity for experiencing a reflective position, thinking that reflection upon our own theoretical and epistemological choices, our social positions and personae - that shape the interpretations we produce - are the basis for the emergence of a more complex, more differentiated, and possibly generalised understanding (Cornish et al. 2007; Valsiner 2007).

The collaborative project in which we participated is a Research and Development Project called DUNES (Dialogic and argUmentative Negotiation Educational Software) which was designed to produce software tools to be used in schools to support the development of argumentative skills. It was composed on the one hand, of Pedagogical partners who are working in the field of education and psychology, and on the other hand, Technical partners who were responsible for the software development. This meeting of two kinds of expertise involves

\footnotetext{
${ }^{1}$ Since we were both participants in the project and observers of the psychosocial dynamics occurring in it, in this paper we assume this two-side position in using the personal pronoun "we". Like ethnographers who try to become members of the group they wish to understand, we take benefit of our inner familiarity with the rules and implicit knowledge that were in use in the project, and try to learn the culture and to account for it "from the inside out".
} 
complementary contributions in order to accomplish the project objectives. The present study describes the construction of this mutual engagement between the different partners, and their construction of new understandings.

We focus our study on diversity in collaboration, and on how and when diversity is made visible, explicit and an object of discussion and negotiation by the project partners themselves. We claim that these moments of tension and negotiation are key points in the elaboration of collaborative work, and help the partners to make explicit - and the researchers to grasp - the psychosocial dynamics at work in the construction of knowledge.

\section{Theoretical Framework for Analysis}

Studies in developmental psychology and education have shown that disagreements and conflicts as well as negotiation and agreements are important processes that enable us to learn and transform practices of doing and knowing (Andriessen et al. 2003; Doise et al. 1975; Perret-Clermont 1980). What are their roles in adults' interactions in managing such a common interdisciplinary research project? Our framework for conceptualizing collaborative production of knowledge is influenced by two theoretical viewpoints: (a) A "community of practice" perspective and (b) activity theory. We present a brief overview of these perspectives and describe their contributions to our object of study.

Community of Practice: Learning to Become a Member of a Community

Working teams can be seen as evolving communities of practices. A community is defined largely by its practices, regulated, explicitly or implicitly, by rules or norms, and the tools that are used to carry them out (see for instance Lave 1991; Wenger 1998).

The main processes at stake for building and maintaining a community are mutual engagement and shared repertoire. Mutual engagement involves all the members of the group working together pursuing a shared goal. It requires the complementary competences of the diverse team members, as in a medical operating team. The development of a shared repertoire is fundamental to maintain and develop the community. It includes routines, words, tools, ways of doing things, stories, gestures, symbols, concepts, and so on, which the community has produced or adopted in the course of its existence and which have become part of its culture. Disagreements, challenges and competition can all be forms of participation in building this culture (Wenger 1998). Collaboration is possible in that it is always negotiated.

Moreover, in entering into a community of practice, people learn to become a competent member of the community. So constructing a new expertise involves learning to belong to a community at the identity level.

This framework emphasizes diversity as a point of departure and a means to develop joint enterprise. As socio-cognitive conflict has been demonstrated as a learning process, Wenger (1998) proposed that disagreement may be a part of the community building process. Moreover, he sheds light on negotiation practices that are considered as constitutive of collaboration in teamwork. These theoretical tools, 
mainly negotiation and shared repertoire as well as entering into a community as an identity building process, are particularly useful to understand the conditions under which collaboration may emerge in an interdisciplinary project like DUNES. We will use them in order to describe how the partners, progressively, if ever, elaborated a kind of "community of practices".

Activity Theory at Work: Systems of Tension and Tensions in Activity Systems

We also draw on activity theory (AT), which considers human activities as systems where knowledge and change develop in collective practices. Based in the work of Vygotsky and Leontiev, this tradition claims that activity is socially mediated: consciousness and meaning are always formed in interactive and collective activity (Leontiev 1978). As a result, the unit of analysis is an activity system, in which the focus is on the interplay between what participants actually do, the objects that motivate their activity, the tools they use, the community to which they belong, rules and division of labor they use in their activity. Tools, objects, rules, communities, and division of labor are social and material resources that both constrain and enable human agency and that mediate relations between participants and the object of their actions (Engeström 1987). A community, a tool, or an individual cannot be theorized in an independent way but must be understood in terms of the historically changing mediated relations of which they are integral and constitutive parts (Roth et al. 2004).

Conflicts, tensions and diversity are intrinsic to activity systems. Several studies in AT (for example in healthcare or within school contexts) have examined these zones of tension by studying competing discourses and practices within a particular social space (Engeström 1993; Gutierrez et al. 1999). These tensions, conflicts and disagreements are here viewed as potential sites of ruptures, innovation and change that bring resources for the participants and for activity development. In our study, we will focus our attention on such points in order to shed light on tensions that appeared in the project and that played the role of opportunities for changes at individual and collective levels.

Thus, AT focuses our attention on several questions: What are the zones of tensions that may lead to learning and development? Between whom do they occur? How are they created? How do they develop? Let us enter into our concrete example, guided by these two theoretical approaches.

\section{Participants and the Project}

The case being presented here is a European Research and Development Project called DUNES. This project was funded under the Fifth Framework Programme of the European Commission (EC). Nine different partners from France, Germany, Greece, Holland, Israel, Sweden, Switzerland, and United Kingdom were represented each by two or more members, who worked together for thirty months from 2002. The main goal of the project was to develop software that can promote argumentative skills. Since the project involved both the development of an ICT tool and its testing and evaluation in educational context, partners with technical 
backgrounds (four firms) and partners with educational and psychological expertise (five university teams) were chosen for this joint enterprise. Technical and Pedagogical partners are involved in the development of the software, "Digalo", 2 but in order to implement and test it in learning settings, many other actors, from different professional fields, such as school authorities, teachers, etc. participated in DUNES.

\section{Methodological Approach}

We attempt to grasp and describe the ways collaboration between teams has been established during this project, and what kinds of learning outcomes were reached. We chose a methodological approach that focuses on (a) the concrete social practices in which the different partners are engaged; (b) the members' perspectives (the analysis does not rely on pre-established categories); (c) examples that we consider as characteristic for describing processes at stake, (d) the way people negotiate the meaning of the situations they live in - as we think that it is in interactive processes that they both contribute to the construction of their contexts and develop new understandings.

In order to enter into the complexity of our object of study, we have made some methodological choices: we have reconstructed the main chronological steps that seem meaningful from the different actors involved. Three steps were identified, following the chronology of the project; they all are strongly shaped by objectives imposed by the EC funders' schedule. The first step is the software development; the second one is its testing in educational contexts, and the third is the analysis of the pupils' learning processes. Within each step, we identified preferential relationships between the different actors involved. We here focus on two main collaborations: the one between Pedagogical and Technical partners, and the one between different Pedagogical teams.

In the following analysis, for each step, we focus on the tension and negotiation zones. We select some extracts as they were carriers of these "tensions" between partners, asking: what are the issues about which tensions develop? How are these tensions taken into account and managed? Finally, for each step, we examine the outcomes in terms of learning: what have the participants - and therefore we as partners-learned from this participation in an interdisciplinary project?

\section{Data Sources}

The data were collected from four different sources: recordings of some of the meeting conversations, archives of electronic communication (e-mails), collective writings of scientific documents (mainly co-constructed through the use of accept/

\footnotetext{
${ }^{2}$ The Technical partners developed a platform, called Oasis, in which the main tool, Digalo, is a graphical editor that allows the users to create and handle argumentative maps. Fed by the users' written contributions, these maps increase through discussion and provide a picture of its evolution-who said what, when, to whom, etc.- -while notifying the argumentative form and structure of the discussion (Schwarz and Glassner 2007).
} 
modify functions of Microsoft Word) and notes during the meetings. The analysis that we develop in the present study is based mainly on documents such as e-mails, 'deliverables' (the division of labor is realised through a work package which ends in a report called a deliverable) and some of their draft versions.

\section{Negotiation and Tensions as Key Points in the Development of the Project}

Our analysis is oriented towards three steps of the project and the main tensions that occur at each one: (1) the software development stage-which involved a high degree of collaboration between Pedagogical and Technical teams; (2) the prototype testing - in which the Pedagogical partners collaborated together and had to agree on how to collaborate with the local partners; and (3) the analysis of the results of the implementation, conducted by the Pedagogical partners. Who is collaborating with whom, for what purpose? What kind of constraints and difficulties do actors face? Under which circumstances? What kind of learning outcomes are developed? These questions will be investigated at each step of the project.

\section{Developing the First Software Prototype}

Two communities were particularly involved in this first step: on the one hand, three Technical teams who had to construct a concrete object, namely the software, and on the other hand, the five Pedagogical teams whose theoretical assumptions on learning and argumentation supported the elaboration of the software affordances.

Concretely it appeared that an important part of the project had to be devoted to the design of the environment, to the neglect of its implementation and testing, not only for technical but also for communication and negotiation reasons. Each group (the Technical and the Pedagogical partners) had their own representations, dreams, and constraints about the object under construction. At the beginning of the project we saw both groups working within their own activity systems, thus with their own vocabulary, privileged mediating tools, objects, rules, community of peers, and division of labor. From the e-mails and the notes we took in several meetings, it is obvious that each group made reference to its own professional repertoire and implicit orientation, either in computer science or in educational fields. For example, difficulties emerged when each community brought their specific concepts: "ontology", an important notion in computer science, was used by the Technical teams, whereas "interaction", "dialogic", "argumentation" were used in the educational field. The Pedagogical teams wanted a flexible, interactive, friendly tool, stressing the importance of permitting the learners, in various educational contexts, to be involved in discursive practices that teachers and researchers could follow and analyze. The Technical teams made reference to a more "product" and "control" oriented way to define argumentation and thus the tool to be developed.

One of the consequences of these differences was that the Pedagogical teams were sometimes considered "dreamers" by the Technical teams, who were, in turn, considered "technocrats" by the Pedagogical teams. This vocabulary revealed some tensions, misunderstandings and difficulties in resolving the problem jointly: how to decide on the affordances of the software in a collaborative way? Progressively, both 
groups found ways to agree on the main basis that allowed the concretisation of one common object: a software tool named Digalo. The moves from a software definition that belonged to one group to another more shared by both groups implied an important work of "translation" and learning of a new language. At the level of the Technical group, who had to elaborate an object from the prerequisites given by the academic partners, taking into account their own technical expertise (and their own representations about argumentation and learning), they had first to understand what was meant by the educational concepts. From the Pedagogical partner side, technical vocabulary and constraints had to be understood as well. This interactive process is illustrated in the following e-mail extracts. The first one is written by a Technical partner and the second by a Pedagogical one:

Extract 1: "On our side [technical group], we are working on a first draft of a general Entity-Relationship schema intended to make a synthesis (at time being) of the concepts introduced by the academic partners so far. This is supposed to represent our understanding of the main "business entities", i.e. the basic concepts manipulated by the system. (...). It shall allow ALL partners to adopt this vocabulary for mapping it to the technical aspects of the system" (29.04.02)

Extract 2: "Thank you for your Fantastic!!! Work and i wish to thank to all of you to your overwhelming efforts to make this eclectic, dialectic, argu-etic... etc...to become real. $\mathrm{i}$ know that this was done in an heroic effort to put almost All of our ideas together and $i$ wish to thank you also for this. I feel that some of us might need a "translation" for some issues which I'll try to write in the coming days. Have the best weekend"(Pedagogical partner, 10.05.02).

This example is a good illustration of what Wenger (1998) called the building of a joint enterprise relying on sharing a repertoire. However in these processes, it is not only a shared repertoire that is co-constructed, but also a sense of belonging to a common group, whose members are part of a same entity-even if the differences between both groups seem obvious. In the Pedagogical partner's message, the borders between the groups are even stressed when he uses the pronoun "you" several times in order to designate the technical groups, and "we" or "us" to refer to the pedagogical partners. It is interesting to notice that he presents himself as a potential mediator between both groups ("I feel that some of us might need a "translation" for some issues which I'll try to write").

The tensions over the definition of the software itself and its creation in this step were mainly concentrated on the fact that we shared neither a common theoretical background nor a previous experience of working together. These tensions, that sometimes took the form of misunderstandings, appeared at first too great to overcome. But through negotiation, translation and mediation we managed to coconstruct a common ground on which we relied to pursue the project. It seems that not only the common contract that linked us with the EC funders played a role but also the sense of belonging that developed during this phase for both communities. This effort to elaborate a sense of belonging is manifest at different occasions like in 
this extract from an e-mail posted by the pedagogical coordinator responding to the news that Digalo (finally) worked:

Extract 3: Dear xxx, Dear all! These are GOOD NEWS!!! We all know how much efforts from all sides (Technical \& Pedagogical) are needed to achieve that! So we are on the right path!! Good luck and Congratulations!! (06.02.03).

We can see the development of a shared repertoire as an important outcome for the whole project and its members. The authors of the final report considered it as so important that they noted it as an added value of the DUNES project:

Extract 4: The cooperation between TECH and PED partners was the driving force at the core of DUNES development, an ongoing give-and-take in which the tool's pedagogical and technological requirements and features became more and more elaborated, more and more refined. (...) This [the overcoming of differences of background and expectations] was done through a process of negotiation and compromise between the partners, involving a convergence of $\mathrm{PED} / \mathrm{TECH}$ expectations, a development of a common language and terminology, and mutual fertilization (p.18).

The building and negotiation of a shared repertoire however did not occur only between the Pedagogical and Technical partners but also between the Pedagogical groups at different moments of the development of the project.

\section{Implementing DUNES in Educational Contexts}

Another important phase of the project was to enter into collaboration with local actors in the educational field in order to test the software in real conditions. The relationships between a Pedagogical team and its local partners were however shaped and structured in the broader frame of the (contractual) relationship of the Pedagogical team with the DUNES project. The project's contractual context shaped the composition of the teams, participants' expectations and pressures under which we worked (see Somekh and Pearson 2002 for a more detailed EC project contractual description).

One of the most important "tensions" to emerge at this step concerned how to define the kind of relationship each Pedagogical team was to construct with the socalled end-users (in fact the teachers). Some partners, making reference to the EC funders' expectations, found that it would be important to formalize the relationships between each team and their 'end-user' partners with contracts. However, it so happened that each team had different "traditions" and institutional educational contexts. This first proposition thus faced the disagreement of some Pedagogical teams who wanted to keep their own ways of working with the teachers without this formal and unusual document. We had therefore to negotiate among the Pedagogical teams and to understand that it was more efficient to manage the collaboration with the teachers differently in each context. These issues were raised for instance in the following e-mails. 


\section{Extract 5: Dear xxx,}

(...) In discussing with $\mathrm{xxx}$ and $\mathrm{xxx}$, we agreed among us about some points and would like to share with you our reflections - and sometimes our surprises (...).

1) During the meeting in Amsterdam (...) we reached a common understanding (or, here in $\mathrm{xxx}$, we thought that we reached a common understanding) about the fact that each pedagogical DUNES partner, due to the specificities of their political, institutional, educational contexts (and discipline, age of the pupils, and so on), would work with their school partners in taking into account these specificities. (...) Moreover, we thought (we are convinced by that) that taking into account these specificities would be an important added value for the project.

The response posted is the following:

\section{Extract 6: Dear xxx}

Thanks for your elaborated and most interesting letter describing xx's approach for introducing DUNES in school. (...) It's true that in the London meeting we've come to different conclusions regarding the process of introducing DUNES in school. It's also true that in Amsterdam we had discussed different directions which somehow meet my previous thoughts (and my present pedagogical beliefs) regarding this issue. On the other hand, we have to bear in mind that we need some "pre conditions" that will include all or most of our end users in the different countries in order to have some "general" evaluation regarding the introduction and the use of DUNES. In a way, we are "trapped" here between two different scientific, philosophical and pedagogical concepts and I think this is where we are in our discussion.

In this e-mail exchange, we can observe a zone of tension: the latter emphasizes the importance of sharing common practices across the countries in order to have some kinds of "comparable" results; the other stresses the necessity to take into account the particularities of each context. Behind these conflicting views about how to collaborate with local partners, it is a definition of a collaborative project that is at stake: each partner doing the same things or each partner adapting their practices to take account of the specificities of their local context? So the tensions at this step emerge at two levels: at the object-oriented level (the object "ways of working with the end-users" is negotiated and transformed during this step), whereas at the coordination and management of the project level, new tools (e.g. ways of communicating and ways of doing research) are negotiated. We took some time to present the specificities of our own educational contexts and our usual ways of working with school authorities. This phase appeared to some as a waste of time but it allowed an understanding of our diversity regarding this issue, and its importance. After stagnation and lack of progress, we eventually decided jointly for each team to adopt their familiar practices. Two teams choose contractual forms and the three other ones did not. In fact, the resolution of this divergence was only in allowing space for diversity.

The difficulties we met at this particular step of the project were in deciding and defining collaboratively, through the question of the end-users, how to work 
together. From initially questioning each others' traditions, we reduced the tensions by accepting diversity in our research practices. Whereas the diversity appeared first as a barrier, it became a resource for intercultural and interdisciplinary work.

This necessity to coordinate and negotiate common goals because of EC constraints gave us opportunities to reflect on and question our own research practices. The main outcomes at this step were that we learnt to share our own research practices making them explicit for the others. These clarifications of our own practices in turn led us to be reflective about our own theoretical, methodological and practical choices. This was possible during this phase of the project as we had already managed to work together and to construct a first common ground, and a sense of belonging to the same community of practices.

\section{Analyzing the Implementation Phase in Usability and Learning Terms}

At this third step of the project, the different Pedagogical partners had to agree on a common ground for the research activity: what would be our guiding research questions? In which terms do we formulate them? These questions have been considered as means of reaching common goals and research outcomes. But in the course of the project these means became objects of tension. To show these movements and transformations, it is interesting to observe the development of the document that was meant to provide this shared understanding. In the first version, the research questions focused mainly on "what is good argumentation?", and were more focused on pedagogical outcomes. Progressively, through a lot of e-mail exchanges, discussions in meetings and co-writing of several documents for the EC funders, it turned into "How can we observe psychological processes at stake in argumentative activities?".

Let us take as an example some extracts of the first version and the last version of the "research question" document (Table 1).

Building a common agreement on how to do research in this project is to be understood within a wider challenge about how to communicate. The questions about how to talk to each other, how to comment on a document written by others, and how to take a joint decision emerged in a quite explicit way in the course of the project. It took time and energy to build these communicative rules among the five groups of Pedagogical partners.

Table 1 Comparison of the first and final versions of some research questions

First version (extracts)

1. Are the functions of the DUNES environment such as "explain", "challenge", "agree", etc. used adequately?

2. To which extent the written dialogue is argumentative?

3. Do participants comply with the roles the role player assigns to them?
Final version (extracts)

1. How can we observe a transformation from opinions to arguments?

2. What kind of uses of the map can we observe?

3. What kind of argumentative practices in the process of elaborating the argumentative map can we observe? 
The following example is extracted from an e-mail exchange between two Pedagogical partners. One of the partners who was in charge of the management of the pedagogical activities wanted to remind their partners that they had to give some feedback about their findings and that it had to be done quickly as the deadline fixed by the coordinator was soon approaching.

Extract 7: 8.9.02 [dunes-all] wake up call to all academic partners

Dear all academic partners

$i$ hope that you had a chance to enjoy and relax in your Summer vacation and that you are back to work with new energies and joy. September has already stepped in and i would like us to continue our discussions regarding the pedagogical preparations and the end-user status in your countries.

As it was a long vacation since our meeting in London i would like to remind you the decisions we all agreed upon as they appear in the minutes(...)i wish to see all of you participating. i need your confirmation for this as well

(...) $\mathrm{i}$ wish us all a continuation of a fruitful thinking and creation according to our DUNES spirit and tradition.

Another Pedagogical partner posted this answer:

Extract 8: 9.9.02 Re: [dunes-all] wake up call to all academic partners Dear xxxx, thank you for your message.

I know that communication across distance and across cultures is not very easy. One way to improve it is - I suppose - to try to solve misunderstandings as soon as they appear. So I want to tell you, in a spirit of friendship, that this message makes me very uneasy. First the title. I can assure you that we are not sleeping (why do you suppose so?) not even academics (why do you single us out?!)... but this is only a minor matter. Jokes is the most difficult to share in foreign languages and I suppose that this is just a joke. More important is the pedagogical/research process. Why should it be so rigid? Why doesn't it build up upon our previous experiences, our discussions with $\mathrm{xxx}$ in preparing DUNES? (...).

This exchange sheds light on two zones of tension: it is not only the content (how to proceed in research work following EC expectations) but the "tone" of the message that are at stake. This message allowed a kind of reflective attitude, in rethinking the way one can talk to each other and for what kinds of objectives. An understanding of the complexity of intercultural communication has developed here.

The outcomes at this step were again gaining a reflective stance but not only to reflect upon one's own research tradition as in the second step but also to develop a reflection on one's own discursive and communicative practices, and to develop one's professional identity as a researcher. We can see that what was meant to be used as a means for doing research collaboratively (the "Research Questions" document) became an object of tension, and led us to redefine new research questions, new ways of researching together and finally new ways to communicate and collaborate. We asked ourselves: What does it mean to engage in an interdisciplinary project as a researcher? How should we take into account the other's perspective without discarding the aim of the project? We learned at a metadiscursive and communicative level as well as an identity level. 


\section{Learning Through Participation in a Collaborative Project}

This analysis of practices of and about collaboration in the course of a "collaborative project" has shown how zones of tension develop and how they can be seen as playing a role in the development of new objects and outcomes of the activity.

The present study extends prior research on collaboration in an interdisciplinary project in two primary ways. First, relying on Wenger's framework and AT analytical tools, we provided a detailed examination of diversity and conflicts as creative nodes of community of practices. It has been our intention through this article to show, focusing on practices, that processes under which such a project evolved are not linear but punctuated with flashbacks, stagnations, changes, disruptions, as for example in the second step around how to define working with end-users. Second, the originality of this work is to study not an already existing community but a new one in the course of its birth and development. We showed that collaboration has to be described rather than to be taken for granted. And the results showed the fact that becoming members of a new community involves several kinds of learning. But what exactly have we learnt? Three different aspects of learning outcomes can be highlighted: (1) learning to become a member of a community in development by co-constructing a shared repertoire (2) learning to generate a new research approach in argumentation and education; (3) learning to reflect upon others' activity and our own.

(1) In the process of developing software, DUNES partners have been engaged in bridging "cultural" gaps by the means of translation, negotiation, and learning of the others' language processes: in order to collaborate in such a project, it seems not enough to be an expert in computer sciences or in educational psychology. For example, in step one two "clans" faced, the "dreamers" against the "technocrats"; but this opposition was probably necessary to begin the construction of a concrete object and to make something real from the imaginative object partners had in mind before its elaboration. In this phase some people played the role of mediator who allowed bridging the gap between the differences that were at first a constraint. One of the most important outcomes has been to understand that it is crucial to elaborate a broad framework in which each team can work according its tradition and institutional contexts. In a sense, we learnt to listen to each other, to take into account the other's viewpoint and way of working even if at first we did not agree or share the same perspective. For example, in step two, we saw that our own traditions of research were too different to adopt the same agenda; so we had to find a way to make them co-exist. But this appeared first as a failure of our collaborative work. It took time and energy to understand that even if we did not use exactly the same procedures, we could still meet the aims of the project. The fact that each team kept developing its own research practices appeared at nearly the end of the project as a gain whereas it was considered as a barrier at the beginning of the second phase. As a consequence, for the DUNES community, collaboration evolved through negotiation of disagreements. Collaborating in a European interdisciplinary project is a time consuming enterprise. However taking time in order to take into account and explicate 
misunderstandings, word meanings, ambiguous positions, and to bring communication towards a meta-level is never a waste of time.

(2) We also learnt about the research practices themselves and the diverse forms they can take. At the beginning, the difficulty of implementing the same methodological approaches in all countries was interpreted as incapacity of the researchers to "collaborate". So, the different partners at first did not feel at ease: we had the impression of not practicing collaborative research the right way. However, the agreement we finally reached, to keep our own tradition rather than trying to standardize our procedure, opened new avenues for an intercultural learning about school practices, relations between researchers and teachers, etc. in the different countries involved in the project.

This complicated process was effective. We developed productive collaborations during and after the project itself: two teams managed to organize an international debate between students through DUNES software, several cases were used and adapted in different educational settings and curricula, collaborative symposia have been organized in international congresses, new European projects have been funded involving some DUNES partners.

(3) We learnt at an individual level and, within teams, at a community level. The long process of developing common research questions, for instance, which would satisfy everybody, can be seen as a means that developed new understandings about the complex activity of doing research collaboratively. But it can also be seen as a challenge that helped us to be more aware of what is a research question that allows describing and analysing argumentative processes. We elaborated new understandings about our research object in its theoretical (thanks to discussions, exchange of bibliography references, collaborative writing from data analysis, etc.), and practical dimensions (how to help teachers to generate argumentation in their class, how to design argumentative activities, how to use Digalo in effective ways, etc.).

In spite of difficulties and constraints, some social competencies have probably been developed, for instance how to listen to and take into account the other's position, to formulate one's own position, to enter into debate. In fact, all these competencies are the very argumentative competencies that we intended to develop in the software's users, the learners.

Participation in such an experience of collaborative work had allowed us to take as object of reflection and study our own meaning-making processes, and our capacity to share and construct new understandings with others, therefore to practice a reflective stance that can be one of the gains of this experience.

It seems that during the participation in a collaborative project, people's understandings, mediating tools and objects co-emerge and co-develop together. As we saw, this movement is not a linear and harmonious one at all: disruption, tensions between groups and people are usual and, through their joint resolution, they may become a resource for generating new meanings and practices. It appears that collaboration sometimes involves accepting not to do the same thing at the same time and that it is often the result of negotiation processes about how to collaborate. 
Acknowledgements This research was carried out in the frame of the DUNES Project. DUNES is a European project coordinated by Professor Baruch Schwarz, Hebrew University of Jerusalem, and funded by the Vth Framework Programme of the European Commission (IST-2001-34153) (www.dunes.gr). We would like to warmly thank all the partners involved in this project and other partners involved in COCASE (Collaborative Case Studies For A European Cultural Psychology) community (funded by European Science Foundation Exploratory Workshop) for their comments and reactions. We are grateful to Tania Zittoun, Flora Cornish and Alex Gillespie for their stimulating invitation. We thank Denis Hilton for his careful English revision of the manuscript.

\section{References}

Akkerman, S., Admiraal, W., Simons, R. J., \& Niessen, T. (2006). Considering diversity: Multivoicedness in international academic collaboration. Culture \& Psychology, 12(4), 461-485.

Andriessen, J., Baker, M., \& Suthers, D. (Eds.) (2003). Arguing to learn: Confronting cognitions in computer-supported collaborative learning environments. Dordrecht: Kluwer.

Cornish, F., Gillespie, A., \& Zittoun, T. (2007). A cultural psychological reflection on collaborative research. Forum: Qualitative Social Research, 8(3), Art. 21. Available at: http://www.qualitativeresearch.net/fqs-texte/3-07/07-3-21-e.htm.

Derry, S., Adams DuRussel, L., \& O’Donnel, A. M. (1998). Individual and distributed cognitions in interdisciplinary teamwork: A developing case study and emerging theory. Educational Psychology Review, 10, 25-56.

Doise, W., Mugny, G., \& Perret-Clermont, A. N. (1975). Social interaction and the development of cognitive operations. European Journal of Social Psychology, 5(3), 367-383.

Engeström, Y. (1987). Learning by expanding: An activity-theoretical approach to developmental research. Helsinki, Finland: Orienta-Konsultit.

Engeström, Y. (1993). Developmental studies of work as a testbench of activity theory: The case of primary care medical practice. In S. Chaiklin \& J. Lave (Eds.), Understanding practice: Perspectives on activity and context (pp. 64-103). Cambridge: Cambridge University Press.

Gutierrez, K. D., Baquedano-Lopez, P., \& Tejeda, C. (1999). Rethinking diversity: Hybridity and hybrid language practices in the third space. Mind, Culture and Activity, 6(4), 286-303.

Lave, J. (1991). Situating learning in communities of practice. In L. Resnick, J. Levine, \& S. Teasley (Eds.), Perspectives on socially shared cognition (pp. 63-84). Hyattsville, MD: American Psychological Association.

Leontiev, A. N. (1978). Activity, consciousness and personality. Englewood Cliffs: Prentice Hall.

Marks, M. A., Burke, C. S., Sabella, M. J., \& Zaccaro, S. J. (2002). The impact of cross-training on team effectiveness. Journal of Applied Psychology, 87, 3-13.

Muller Mirza, N. (2005). Psychologie culturelle d'une formation d'adulte. L'île aux savoirs voyageurs. Paris: L'Harmattan.

Perret-Clermont, A. N. (1980). Social interaction and cognitive development in children. London: Academic.

Roth, W. M., Tobin, K., Elmensky, R., Carambo, C., McKnight, Y., \& Beers, J. (2004). Re/making identities in the praxis of urban schooling: A cultural historical perspective. Culture, Mind and Activity, 11(1), 48-69.

Schwarz, B., \& Glassner, A. (2007). Designing CSCL argumentative environments for broadening understanding of the space of debate. In R. Säljö (Ed.). ICT and the transformation of learning practices. New York: Pergamon.

Somekh, B., \& Pearson, M. (2002). Intercultural learning arising from pan-European collaboration: A community of practice with a 'hole in the middle'. British Educational Research Journal, 28(4), $485-502$.

Valsiner, J. (2007). Culture in minds and societies: Foundations of cultural psychology. New Delhi: Sage.

Wenger, E. (1998). Communities of practice: Learning, meaning and identity. Cambridge: Cambridge University Press.

Wertsch, J., \& Toma, C. (1995). Discourse and learning in the classroom: A sociocultural approach. In L. P. Steffle \& J. Gale (Eds.), Constructivism in education (pp. 159-175). Hilsdale, NJ: Erlbaum. 
Valérie Tartas is a lecturer and researcher in developmental psychology within the Cognition, Communication and Development Laboratory at the University of Toulouse 2, France. Her principal interest topics are about the relationship between social context, mediation tools and learning. She is interested in socio-cognitive development through the appropriation of cultural tools by children with a special interest in the development of children's thinking and language. She has recently taken part in research about learning through argumentation supported by ICT tools in two European projects: Dunes (Dialogic and argUmentative Negotiation Educational Software, 2002-2004) and Escalate (Enhancing SCience Appeal in Learning through Argumentative interaction, 2006-2007).

Nathalie Muller Mirza is a Doctor in Humanities and Human Sciences (Psychology). She is a lecturer and researcher in Psychology and Education in the University of Neuchâtel. Her main research foci are on psychosocial processes in learning settings, in and out of school and in diverse cultural contexts. Since 2001, she is particularly interested in argumentation as a tool for learning in classroom and training contexts from the perspective of a socio-cultural psychology; she is involved in several European projects (DUNES, ESCALATE; coordinated by the Hebrew University of Jerusalem) aiming at developing and analysing the role of argumentative software in learning. She is part of the Swiss Virtual Campus 'Argumentum' and gives lectures on argumentation and its psychosocial dimensions in social contexts. Her publications include: Muller, N. \& Perret-Clermont, A.-N. (1999). Negotiating identities and meanings in construction of knowledge. Analysis of interactions in a specific context, a "Network of exchange of knowledge". In J. Bliss, R. Säljö \& P. Light (Eds.). Learning sites. Social and technological resources for learning (pp. 47-61). Oxford : Pergamon; Muller Mirza, N., Tartas, V., Perret-Clermont, A.-N. \& de Pietro, J.-F. (2007, to appear). Using graphical tools in a phased activity for enhancing dialogical skills: an example with DUNES. IjCSCL Special issue "Using argument graphs to support collaborative learning" (coordinated by D. Suthers, J. Andriessen \& M. Baker). 